\title{
Cross-Cultural Adaptation and Preliminary Validation of the European Spanish Version of the Developmental Coordination Disorder Questionnaire (DCDQ-ES)
}

\author{
Rebeca Montes-Montes ${ }^{\mathrm{a}}$; Laura Delgado-Lobete ${ }^{\mathrm{b}}$; Javier Pereira ${ }^{\mathrm{a}}$; Thais Pousada ${ }^{\mathrm{a}}$ \\ a TALIONIS Research Group, Research Center of the Galician University System, Center for Information and \\ Communications Technology Research, Universidade da Coruña, A Coruña, Spain. \\ ${ }^{b}$ Health Integration and Promotion Research Unit (Integra Saúde), Faculty of Health Sciences, Universidade \\ da Coruña, A Coruña, Spain.
}

\begin{abstract}
Importance: The Developmental Coordination Disorder Questionnaire (DCDQ) is the most widely used measure of difficulties with activities of daily living and academic performance in children with developmental coordination disorder, but this tool has not been adapted for use in Spain.

Objective: To translate and cross-culturally adapt the DCDQ into European Spanish (DCDQ-ES) for use in assessing motor coordination in Spanish children.

Design: Cross-cultural adaptation and preliminary validation study.

Setting: Community and mainstream schools in Spain.

Participants: A committee of five experts oversaw the cross-cultural adaptation process. A community-based convenience sample of 31 parents of children ages 5-14 yr was used to test the comprehensibility of the DCDQ-ES. Preliminary reliability was tested with 35 randomly selected parents of children ages 6-12 yr.

Outcomes and Measures: The DCDQ was translated into European Spanish and cross-culturally adapted following international guidelines. Comprehensibility was assessed using cognitive debriefing interviews. The final version of the DCDQ-ES was used for the reliability analysis.

Results: Cultural relevance and equivalence and idiomatic differences between the DCDQ and DCDQ-ES were evaluated. Comprehensibility analysis led to minor modifications that facilitated comprehension and interpretation. Internal consistency and homogeneity of the DCDQ-ES were good (Cronbach's $\alpha=.857$, corrected item-total correlations $=.268-.692$ ).

Conclusions and Relevance: The DCDQ-ES is conceptually and semantically equivalent to DCDQ and was successfully cross-culturally adapted for the European Spanish context. Preliminary data suggest that the DCDQ-ES is a reliable measure of motor coordination in Spanish children.

What This Article Adds: This study provides evidence of the cultural equivalence of the DCDQ-ES for use with Spanish children. Occupational therapists in Spain can use the DCDQ-ES to evaluate children's motor coordination difficulties in everyday activities.
\end{abstract}


Developmental coordination disorder (DCD) is a motor neurodevelopmental condition that affects an estimated 5\%-6\% of school-age children (American Psychiatric Association, 2013; Blank et al., 2019). This disorder has a significant impact on executive function, daily life activities, and academic achievement throughout adolescence and adulthood. Therefore, DCD should be addressed as soon as motor coordination difficulties are identified during development (Bernardi et al., 2018; Delgado-Lobete et al., 2019; Harrowell et al., 2018; Magalhães et al., 2011; Wilson et al., 2013).

One of the most widely recognized and validated tools used in screening for and assessing children with DCD is the Developmental Coordination Disorder Questionnaire (DCDQ; Blank et al., 2019; Wilson et al., 2009). This 15-item parent-report questionnaire is designed to assess motor coordination in children ages 5-15 yr. The DCDQ has been successfully cross-culturally adapted for several languages and contexts, and studies have demonstrated that these adaptations are reliable and valid for screening for DCD in children (Caravale et al., 2014; Kennedy-Behr et al., 2013; Martini et al., 2011; Nowak, 2016; Patel \& Gabbard, 2017; Prado et al., 2009; RayKaeser et al., 2015).

A Latin American Spanish version of the DCDQ is available (Salamanca et al., 2012), but its linguistic and cultural characteristics may not match European Spanish idioms or the social context of Spanish children. Latin American Spanish differs from European Spanish in many ways in terms of word meaning, morphology, phonology, and cultural expressions (Otheguy \& Zentella, 2000). When cross-culturally adapting a measure for a different context, it is necessary to consider both semantic and contextual aspects to ensure equivalence of results; a European Spanish adaptation of the DCDQ (DCDQ-ES) that fits the context of Spanish children is therefore necessary. The aims of this study were to translate and cross-culturally adapt the DCDQ for use in Spain and conduct a preliminary assessment of reliability with a Spanish population.

\section{Method}

\section{Translation}

We obtained approval to develop a translation and cross-cultural adaptation of the DCDQ from the developer, Brenda Wilson. Approval for this study was granted by the Autonomic Research Ethics Committee of Galicia (Code 2017/167). We followed the same six-stage process proposed by Beaton et al. (2000; Figure 1) that was used for other cross-cultural adaptations of this questionnaire.

The DCDQ was independently translated by a Spanish occupational therapist (the second author, Delgado-Lobete) and a professional translator with a background in English literature and education. The Spanish occupational therapist was familiar with the DCDQ and had a background in research on DCD (Translator 1), whereas the professional translator was unfamiliar with the DCDQ and with DCD (Translator 2). The two translators and the main researcher (the first author, Montes-Montes) compared the two translations, T1 and T2, and produced a version of the DCDQ that synthesized elements of both translations. This version was independently back translated into English by two bilingual translators who were unfamiliar with the DCDQ and whose first language was English, to produce BT1 and BT2. 
Figure 1.

Process of cross-cultural adaptation of the Developmental Coordination Disorder Questionnaire into European Spanish. Note. $\mathrm{DCD}$ = developmental coordination disorder; DCDQ = Developmental Coordination Disorder Questionnaire.

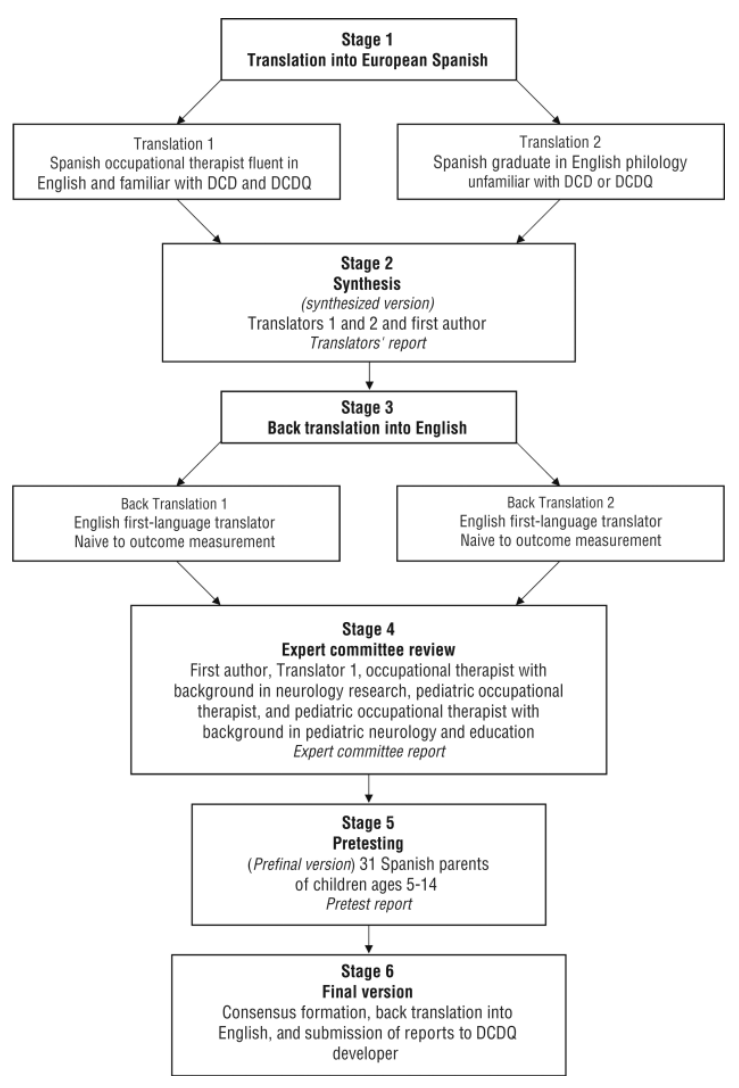

Expert Committee Review and Pretest of Cultural Relevance, Equivalence, and Comprehensibility

An expert committee reviewed and compared T1, T2, the synthesized version, BT1, and BT2 to determine cultural and idiomatic equivalence between the synthesized translation and the original English DCDQ. This committee included the first author (Montes-Montes), Translator 1 (the second author, Delgado-Lobete), a pediatric occupational therapist, an occupational therapist with a background in neurology research (the fourth author, Pousada), and an occupational therapist with a background in pediatric neurology and education. A modified, prefinal version of the DCDQ-ES was produced after the expert committee review.

The prefinal version was tested with a community-based convenience sample of 31 parents of children ages 5-14 yr. The parents were recruited from previously contacted schools located in four different regions of Spain, and they had varied educational and occupational backgrounds. One participant had a child diagnosed with attention deficit hyperactivity disorder, and the rest had children who were considered typically developing. All parents were informed about the purpose of the study and provided informed consent.

To evaluate the cultural relevance, equivalence, and comprehensibility of the prefinal version of the DCDQ-ES, we used the Three-Step Test-Interview (TSTI), a cognitive interview approach consisting of three consecutive phases: think aloud, follow-up, and validation (Hak et al., 2008). 
The interviews were not transcribed because any potential issues were addressed during the interview. The first author (Montes-Montes) led the interviews and wrote down any difficulties expressed by the participants, as well as any nonverbal expression suggesting that some aspects of the DCD-ES were unclear in order to clarify or discuss any potential issues. We used inquiry, paraphrase, exposition, and exemplification to detect difficulties with semantics and grammar (semantic equivalence), interpretation of colloquialisms (idiomatic equivalence), and familiarity with the activities described in the prefinal version (experimental equivalence). As recommended, if $20 \%$ (i.e., $\geq 6$ ) of the participants found an item difficult to interpret or understand, we revised and reworded that item (Sousa \& Rojjanasrirat, 2011).

After rewording the problematic items, we produced the final version of the DCDQ-ES and recorded all stages of the cross-cultural adaptation process. This final version was back translated into English and submitted to the DCDQ's original developer (Wilson), who analyzed the written report of the cross-cultural adaptation process and approved the final version of the DCDQ-ES.

\section{Preliminary Reliability Evaluation}

A second randomly selected sample of Spanish parents was used to evaluate the reliability of the DCDQ-ES. Thirty-five parents of typically developing children ages 6-12 yr attending a randomly selected mainstream school in A Coruña (a city in northern Spain) completed the DCDQ-ES (mothers $=80.0 \%$; children's average age $=9.00 \pm 2.03 \mathrm{yr}$; girls $=51.4 \%$ ). All parents answered the questionnaire anonymously in their home and sent it back to the school, where the first author collected them. Reliability was determined through analysis of the internal consistency and homogeneity of the completed questionnaires.

\section{Data Analysis}

The first author analyzed the cognitive interviews conducted for the prefinal version of the DCDQ-ES to determine whether participants' comments reflected semantic, idiomatic, or experimental difficulties. Chi-square tests were used to explore differences in equivalence between translations. Internal consistency was estimated using Cronbach's $\alpha$, with values $>.80$ considered good. Homogeneity of the items was assessed using corrected item-total correlations, with values $>.20$ considered acceptable (Eremenco et al., 2005). All statistical analyses were conducted using IBM SPSS Statistics Version 20 (IBM Corp., Armonk, NY) and EPIDAT 3.1 (Department of Epidemiology, 2006).

\section{Results}

\section{Initial Equivalence Evaluation}

Both translators evaluated the semantic and conceptual equivalence of the two independent and synthesized translations of the DCDQ-ES; results are summarized in Table 1. The translators agreed on the equivalence of $66.7 \%$ of items across the two translations.

After reviewing the synthesized translation, the translators considered all items to be either totally $(86.7 \%$, 95\% confidence interval [CI] [69.3, 96.2]) or conceptually $(13.3 \%$, 95\% CI [3.8, 30.7]) equivalent. The two translators' equivalence evaluations did not differ significantly $\left(\chi^{2}=\right.$ $0.27, \mathrm{p}=.605)$. 
Table 1.

Equivalence Evaluation of the Independent and Synthesized Translations Into European Spanish and Pretest Results

\begin{tabular}{|c|c|c|c|c|c|c|c|c|}
\hline \multirow{3}{*}{ Item } & \multicolumn{4}{|c|}{ Equivalence Evaluation ${ }^{\underline{a}}$} & \multirow{2}{*}{\multicolumn{4}{|c|}{ Results of Pretest With Parents $(N=31)^{\underline{b}}$}} \\
\hline & \multicolumn{2}{|c|}{ Independent Translations } & \multicolumn{2}{|c|}{ Synthesized Translation } & & & & \\
\hline & Translator 1 & Translator 2 & Translator 1 & Translator 2 & Comments & $n(\%)$ & Difficulty in Understanding? & Reworded in Final Version? \\
\hline 1 & A & A & A & A & SE & $6(19.4)$ & No & No \\
\hline 2 & B & A & A & A & No & - & - & - \\
\hline \multirow{2}{*}{3} & \multirow{2}{*}{ B } & \multirow{2}{*}{ B } & \multirow{2}{*}{ A } & \multirow{2}{*}{ A } & IE & $5(16.1)$ & \multirow{2}{*}{ No } & \multirow{2}{*}{ No } \\
\hline & & & & & EE & $1(3.2)$ & & \\
\hline 4 & B & B & A & A & IE & $12(38.7)$ & No & Yes \\
\hline 5 & A & A & A & A & No & - & - & - \\
\hline 6 & $\mathrm{C}$ & B & $\mathrm{B}$ & A & No & - & - & - \\
\hline 7 & A & A & A & A & No & - & - & - \\
\hline 8 & B & A & B & A & No & - & - & - \\
\hline 9 & B & A & B & A & SE & $5(16.1)$ & Yes & Yes \\
\hline 10 & A & A & A & A & No & - & - & - \\
\hline 11 & A & A & A & A & No & - & - & - \\
\hline 12 & A & B & A & B & $\mathrm{SE}$ & $5(16.1)$ & No & No \\
\hline 13 & A & A & A & A & SE & $2(6.5)$ & No & No \\
\hline 14 & B & B & A & A & SE & $6(19.4)$ & Yes & Yes \\
\hline 15 & B & B & A & A & SE & $3(9.7)$ & Yes & Yes \\
\hline
\end{tabular}

${ }^{\mathrm{a}} \mathrm{A}=$ item is conceptually and semantically equivalent; $\mathrm{B}=$ item is conceptually equivalent; $\mathrm{C}=$ item is not equivalent. ${ }^{\mathrm{b}} \mathrm{n}=$ number of participants who commented on the item; - = not applicable; $\mathrm{EE}=$ comments on experimental equivalence; $\mathrm{IE}=$ comments on idiomatic equivalence; $\mathrm{SE}=$ comments on semantic equivalence. 


\section{Pretest Evaluation of Cultural Relevance, Equivalence, and Comprehensibility}

During the expert committee review, six items were extensively discussed and modified. In Item 4, the Spanish term zona de juegos was modified to zona de juego to include every possible play area. Items $6,7,8,9$, and 15 described motor planning-related activities and were challenging to reword in a way that accurately retained the original meaning. Brief examples were added to these five items to facilitate understanding and interpretation. In addition, Items 14 and 15 were reworded with positive phrasing to avoid possible misunderstandings.

In testing the prefinal version of the DCDQ-ES, all 31 parents (Table 2) displayed good understanding of the items, but they reported difficulties with or made recommendations for eight of the items (results are summarized in Table 1). Most of the comments referred to minor semantic or idiomatic concerns. However, one parent reported difficulty with experimental equivalence for Item 3, which addresses children's performance while playing sports (this issue is explored in the Discussion). According to the participants, the instructions were easy to understand, and the activities were familiar and easy to recall.

Table 2. Sociodemographic Characteristics of Pretest Participants $(\mathrm{N}=31)$

\begin{tabular}{ll}
\hline Characteristic & $\boldsymbol{n}(\%)$ or $\boldsymbol{M} \pm \boldsymbol{S D}$ \\
\hline $\begin{array}{l}\text { Child age, yr } \\
\text { Role }\end{array}$ & $8.2 \pm 2.2$ \\
$\quad$ Mother & $18(58.1)$ \\
$\quad$ Father & $13(41.9)$ \\
Region of Spain & \\
$\quad$ A Coruña (northwest) & $5(16.1)$ \\
$\quad$ Asturias (north) & $22(71.0)$ \\
Valladolid (central) & $2(6.5)$ \\
$\quad$ Madrid (central) & $2(6.5)$ \\
Education & \\
First stage of high school & $1(3.2)$ \\
High school & $8(25.8)$ \\
Technical or community college & $13(41.9)$ \\
University & $9(29.0)$ \\
\hline
\end{tabular}

Note. $\mathrm{M}=$ mean; $\mathrm{SD}=$ standard deviation .

Results from the TSTI evaluation of the pretest led to additional changes in four items. Items 9, 14, and 15 underwent structural changes, and brief examples were included using terms selected to better capture the original meaning of the items rather than the direct Spanish translations. Although Item 4 was easy to understand and interpret, participants reported that it would sound more natural if it were expressed in the indicative mood instead of the subjunctive mood. No differences in reported cultural equivalence or comments were found according to parents' region, indicating that items reflect common activities for the majority of participants from northern and central Spain. 


\section{Reliability}

The DCDQ-ES showed good internal consistency, with a Cronbach's $\alpha$ of .857 (Table 3). The $\alpha$ coefficient did not significantly increase when items were individually removed, indicating that no single item was problematic. Corrected item-total correlations were good (range $=.268-.692$ ).

Table 3. Internal Consistency and Homogeneity of the DCDQ-ES

\begin{tabular}{lll}
\hline Item & Cronbach's $\boldsymbol{\alpha}$ If Item Is Deleted $^{\mathbf{a}}$ & Corrected Item-Total Correlation $^{-}$ \\
\hline 1 & .849 & .482 \\
2 & .854 & .377 \\
3 & .854 & .370 \\
4 & .849 & .485 \\
5 & .857 & .310 \\
6 & .859 & .268 \\
7 & .849 & .476 \\
8 & .850 & .461 \\
9 & .845 & .561 \\
10 & .838 & .692 \\
11 & .840 & .632 \\
12 & .846 & .532 \\
13 & .840 & .627 \\
14 & .837 & .692 \\
15 & .853 & .435 \\
\hline
\end{tabular}

Note. DCDQ-ES = European Spanish version of the Developmental Coordination Disorder Questionnaire. ${ }^{a}$ Total Cronbach's $\alpha=.857$.

\section{Discussion}

The aim of this study was to translate and culturally adapt the DCDQ into European Spanish and evaluate its cultural relevance, equivalence, and comprehensibility with Spanish parents of children ages 5-15 yr. We produced the translation by synthesizing two independent Spanish translations into a prefinal version, testing the prefinal version, and incorporating the results into the final version. We then evaluated the reliability of the final version in assessing motor coordination of Spanish children.

\section{Translation Process}

The translators agreed that the synthesized version better preserved the intent of the original DCDQ than the individual translations and that it reflected the linguistic and cultural characteristics of the European Spanish context. Most processes recommended for translating and cross-culturally adapting questionnaires incorporate generating a synthesized translation from independent translations. However, many studies do not use this procedure and instead use a single translation, likely because it involves less time and fewer resources (Beaton et al., 2000; Sousa \& Rojjanasrirat, 2011). In our study, analysis of the two translations provided an opportunity for the translators and reviewers to engage in debate to uncover relevant information and challenges that would otherwise have gone unnoticed. The high degree of agreement among the translators and reviewers on the equivalence of the synthesized version reflects improvements in the overall quality of the DCDQ-ES. Thus, this study supports the use of two or more independent translations in the cross-cultural adaptation of questionnaires. 
We modified Items 14 and 15, which were negatively worded in the original, to use positive phrasing. As a result, the parents reported that the items were more comprehensible and enabled them to provide more accurate information. Difficulty in comprehending these two items was reported in studies for other European adaptations of the DCDQ, in which it was necessary to reword the items to facilitate interpretation (Kennedy-Behr et al., 2013; Ray-Kaeser et al., 2015).

\section{Cultural Relevance and Equivalence}

Overall, the parents were familiar with the activities described in the DCDQ-ES. Slight modifications were made to some items to better fit the Spanish context; for instance, the expression "bull in a china shop" was replaced with the Spanish equivalent "elefante en una cacharrería," or "elephant in a glassware shop." Other cross-cultural adaptations of the DCDQ have made modifications to reflect cultural differences in common, everyday activities (Girish et al., 2015; Ray-Kaeser et al., 2015).

Although the parents were familiar with the activities described in the DCDQ-ES, some reported difficulty recalling their children's performance in sports-related activities for Item 3. Working parents in particular reported that they rarely had the opportunity to watch their children play outside; most families in Spain have two working parents, and parent-child time is influenced by Spanish family traditions and child-unfriendly work schedules (Gracia \& Garcia-Roman, 2017; Gracia \& Kalmijn, 2016). Parents are usually more involved in children's academic activities during the week and are able to watch them play outdoor sports only on weekends (Gracia \& Garcia-Roman, 2017). Minor difficulties in cross-cultural adaptation of Item 3 were previously reported in the literature, but overall the parents in our sample reported adequate cultural equivalence of this item (Prado et al., 2009; Salamanca et al., 2012).

\section{Idiomatic Difficulties}

Many of the semantic modifications during the expert committee review and pretest process were made to ensure that the items were in line with the original intended meaning in English. Items assessing motor planning actions involving organized and precise activities had to be reworded and brief examples added to facilitate an interpretation closer to the original one (e.g., separating stroke pressure from grasp tightness when writing or drawing for Item 9). Other crosscultural adaptations of the DCDQ also found minor issues regarding unexpected meanings of motor precision on these items (Ray-Kaeser et al., 2015; Salamanca et al., 2012).

The think-aloud phase during the TSTI process revealed that some parents found Items 9, 14, and 15 to be oddly expressed and difficult to interpret. Item 15 was the most discussed item during the expert committee review because the direct translations of slouch and fall out did not adequately reflect the intended meaning of these terms. Although most parents were able to give an adequate example of the activities described in these items, we decided to reword them and provide additional information to facilitate comprehension and interpretation. Idiomatic difficulties were also found in development of the Latin American Spanish version of the DCDQ; Items 14 and 15 were especially challenging to adapt because of the difficulty in translating them to Latin American Spanish, and the items were reworded to positive phrasing and adjusted to the Colombian context (e.g., "bull in a china shop" was deleted from Item 14; Salamanca et al., 2012). 


\section{Reliability}

The preliminary data on reliability of the DCDQ-ES suggest good internal consistency and homogeneity. All of the DCDQ cross-cultural adaptations have demonstrated excellent psychometric properties, including internal consistency and homogeneity similar to those of the present study (Caravale et al., 2014; Kennedy-Behr et al., 2013; Martini et al., 2011; Nowak, 2016; Patel \& Gabbard, 2017; Prado et al., 2009; Ray-Kaeser et al., 2016).

\section{Limitations and Future Research}

The main limitation of this study is that all participants came from northwestern and central regions of Spain, and thus it may be possible that a few minor linguistic and cultural aspects of the questionnaire were not addressed. Some southern, northeastern, and northwestern regions of Spain speak different Spanish dialects, but all Spaniards are taught standard (Castilian) Spanish as the main language. When adapting questionnaires for use in Spain, researchers typically translate them into standard Spanish because it is the main language in Spain and is understood and spoken in the entire country. Therefore, the DCDQ-ES is usable in all of Spain.

Another limitation is the small sample used to evaluate the reliability of the DCDQ-ES. Some authors have suggested that a sample size of 15-30 is sufficient to provide preliminary evidence for internal consistency in health status questionnaires (Eremenco et al., 2005), and this study provided preliminary data from 35 participants. However, additional studies should use larger samples.

\section{Implications for Occupational Therapy Practice}

The findings of this study have the following implications for occupational therapy practice:

- Motor coordination skills are closely associated with occupational performance and participation. A well-validated and cross-culturally adapted tool such as the DCDQ-ES can enable early detection of motor coordination difficulties in children with potential DCD.

- Occupational therapy practitioners working in clinical practice or schools in Spain or with clients from Spain can use the DCDQ-ES to assess a broad range of daily activities in children with motor coordination difficulties.

- This study provides preliminary evidence of reliability of the DCDQ-ES for use with children ages 6-12. The DCDQ-ES is the first cross-culturally adapted tool available in European Spanish for assessing children's motor coordination during everyday activities.

\section{Conclusion}

Our results show that the European Spanish version of the DCDQ is equivalent to the original English DCDQ and is culturally appropriate for use with the Spanish population. This study highlights the need for a rigorous and thorough process in adapting measures for new cultural contexts and populations. Preliminary data suggest that the DCDQ-ES has good reliability, and further testing is required to establish its validity in assessing Spanish children. 


\section{Acknowledgments}

We thank the original author of the DCDQ, Brenda Wilson, for granting permission to translate and cross-culturally adapt it into European Spanish. We also thank the translators, expert committee members, and parents who participated in this study. This research was partially supported by the Galician Innovation Agency through the Pequeña y Mediana Empresa Connect Program (3rd ed.; IN852A 2016/10), assistance for the consolidation and structuring of competitive research of the Galician University System of the Xunta de Galicia (R2014/039), and a grant for Singular Research Centers (ED431G/01) endowed by the European Union's European Regional Development Fund. The authors have no interests that might be perceived as posing a conflict or bias.

\section{References}

American Psychiatric Association. (2013). Diagnostic and statistical manual of mental disorders (5th ed.). Arlington, VA: American Psychiatric Publishing.

Beaton, D. E., Bombardier, C., Guillemin, F., \& Ferraz, M. B. (2000). Guidelines for the process of cross-cultural adaptation of self-report measures. Spine, 25, 3186-3191.

Bernardi, M., Leonard, H. C., Hill, E. L., Botting, N., \& Henry, L. A. (2018). Executive functions in children with developmental coordination disorder: A 2-year follow-up study. Developmental Medicine and Child Neurology, 60, 306-313

Blank, R., Barnett, A. L., Cairney, J., Green, D., Kirby, A., Polatajko, H., . . Vinçon, S. (2019). International clinical practice recommendations on the definition, diagnosis, assessment, intervention, and psychosocial aspects of developmental coordination disorder. Developmental Medicine and Child Neurology, 61, 242-285.

Caravale, B., Baldi, S., Gasparini, C., \& Wilson, B. N. (2014). Cross-cultural adaptation, reliability and predictive validity of the Italian version of Developmental Coordination Disorder Questionnaire (DCDQ). European Journal of Paediatric Neurology, 18, 267-272.

Delgado-Lobete, L., Santos-del-Riego, S., Pértega-Díaz, S., \& Montes-Montes, R. (2019). Prevalence of suspected developmental coordination disorder and associated factors in Spanish classrooms. Research in Developmental Disabilities, 86, 31-40

Department of Epidemiology. (2006). EPIDAT 3.1: Epidemiological analysis from tabulated data [Computer software]. A Coruña, Spain: Dirección Xeral de Saúde Pública.

Eremenco, S. L., Cella, D., \& Arnold, B. J. (2005). A comprehensive method for the translation and cross-cultural validation of health status questionnaires. Evaluation and the Health Professions, 28, 212-232.

Girish, S., Raja, K., \& Kamath, A. (2015). Translation of revised version of Developmental Coordination Disorder Questionnaire (DCDQ’07) into Kannada-Results of validation. Asia Pacific Disability Rehabilitation Journal, 26, 82-100

Gracia, P., \& Garcia-Roman, J. (2017). Parents' nonstandard work hours and children's time use in Spain: The intersection of parental work schedules, gender and education. SSRN.

Gracia, P., \& Kalmijn, M. (2016). Parents' family time and work schedules: The split-shift schedule in Spain. Journal of Marriage and Family, 78, 401-415.

Hak, T., van der Veer, K., \& Jansen, H. (2008). The Three-Step Test-Interview (TSTI): An observational instrument for pretesting self-completion questionnaires. Survey Research Methods, 2, 143-150.

Harrowell, I., Hollén, L., Lingam, R., \& Emond, A. (2018). The impact of developmental coordination disorder on educational achievement in secondary school. Research in Developmental Disabilities, 72, 13-22.

Kennedy-Behr, A., Wilson, B. N., Rodger, S., \& Mickan, S. (2013). Cross-cultural adaptation of the Developmental Coordination Disorder Questionnaire 2007 for German-speaking countries: DCDQ-G. Neuropediatrics, 44, 245-251.

Magalhães, L. C., Cardoso, A. A., \& Missiuna, C. (2011). Activities and participation in children with developmental coordination disorder: A systematic review. Research in Developmental Disabilities, 32, 1309-1316. 
Martini, R., St-Pierre, M. F., \& Wilson, B. N. (2011). French Canadian cross-cultural adaptation of the Developmental Coordination Disorder Questionnaire '07: DCDQ-FC. Canadian Journal of Occupational Therapy, 78, 318-327.

Nowak, A. (2016). Cross-cultural adaptation of the Developmental Coordination Disorder Questionnaire (DCDQ’07) for the population of Polish children. Biomedical Human Kinetics, 8, 17-23.

Otheguy, R., \& Zentella, A. C. (2000). Spanish in New York: Language contact, dialectal leveling, and structural continuity. New York: Oxford University Press.

Patel, P., \& Gabbard, C. (2017). Adaptation and preliminary testing of the Developmental Coordination Disorder Questionnaire (DCDQ) for children in India. Physical and Occupational Therapy in Pediatrics, 37, 170-182

Prado, M. S. S., Magalhães, L. C., \& Wilson, B. N. (2009). Cross-cultural adaptation of the Developmental Coordination Disorder Questionnaire for Brazilian children. Brazilian Journal of Physical Therapy, 13, 236-243.

Ray-Kaeser, S., Satink, T., Andresen, M., Martini, R., Thommen, E., \& Bertrand, A. M. (2015). European-French cross-cultural adaptation of the Developmental Coordination Disorder Questionnaire and pretest in French-speaking Switzerland. Physical and Occupational Therapy in Pediatrics, 35, 132-146.

Ray-Kaeser, S., Thommen, E., Martini, R., \& Bertrand, A. M. (2016). Validation transculturelle du Developmental Coordination Disorder Questionnaire '07 à la population francophone d'Europe: Le QTAC-FE [Cross-cultural validation of the Developmental Coordination Disorder Questionnaire '07 for the Francophone population in Europe: The QTAC-FE]. Revue Francophone de Recherche en Ergothérapie, 2, 6-20.

Salamanca, L. M., Naranjo, M. M. C., \& González, A. P. (2012). Traducción al español del Cuestionario para Diagnóstico de Trastorno del Desarrollo de la Coordinación [Spanish translation of the Developmental Coordination Disorder Questionnaire]. Revista Ciencias de la Salud, 10, 195-206.

Sousa, V. D., \& Rojjanasrirat, W. (2011). Translation, adaptation and validation of instruments or scales for use in cross-cultural health care research: A clear and user-friendly guideline. Journal of Evaluation in Clinical Practice, 17, 268-274

Wilson, B. N., Crawford, S. G., Green, D., Roberts, G., Aylott, A., \& Kaplan, B. J. (2009). Psychometric properties of the revised Developmental Coordination Disorder Questionnaire. Physical and Occupational Therapy in Pediatrics, 29, 182-202.

Wilson, P. H., Ruddock, S., Smits-Engelsman, B., Polatajko, H., \& Blank, R. (2013). Understanding performance deficits in developmental coordination disorder: A meta-analysis of recent research. Developmental Medicine and Child Neurology, 55, 217-228 